\title{
Unitary relativistic quantum theory.
}

\section{Eliade Stefanescu*}

Advanced Studies in Physics Centre of the Romanian Academy, Academy of Romanian Scientists Bucharest, Romania

\begin{abstract}
We consider a quantum particle as a wave packet, and find that the group velocities in the coordinate and momentum spaces are in agreement with the Hamilton equations only when the Lagrangian is considered in the time dependent phases, instead of the Hamiltonian in the conventional forms of these waves as solutions of the Schrödinger equation. We define a relativistic quantum principle, and derive a wave equation for a relativistic quantum particle, the relativistic kinematics and dynamics of the particle waves, the Maxwell equations and the Lorentz force of a field interacting with the particle waves, the relativistic transform of such a field, and the spin as a characteristic of the particle waves. We consider a quantum particle as a distribution of conservative matter propagating according to the General Theory of Relativity. We obtain the dynamics of this matter in a gravitational field, the propagation in plane waves perpendicular to geodesic tracks, and equations of conservation.
\end{abstract}

Keywords: Quantum particle, Wave packet, Group velocity, Lagrangian, Hamiltonian, Maxwell equations, Spin, metric tensor, Covariant derivation.

\section{Introduction}

We perceive the physical world as a collection of objects, in a three-dimensional space of coordinates defined by a position vector $\vec{r}=x \overrightarrow{1}_{x}+y \overrightarrow{1}_{y}+z \overrightarrow{1}_{z}$, and in time, defined by a scalar $t$ [1-5]. For a matter object we define an inertial property called mass, $M_{0}$ and a dynamic quantity as the product of the mass with the velocity, $\vec{v}=\frac{\mathrm{d} \vec{r}}{\mathrm{~d} t}$, called momentum, $\vec{p}=M_{0} \vec{v}$. We also define a conservative quantity, called energy,

$$
E=H_{0}(\vec{r}, \vec{p})=T(\vec{p})+U(\vec{r})
$$

as a sum of the kinetic energy $T(\vec{p})$, which depends on momentum, and the potential energy $U(\vec{r})$, which depends on the coordinates [6-8]. From the conservation condition:

$$
\frac{\mathrm{d} E}{\mathrm{~d} t}=\frac{\partial H_{0}}{\partial \vec{r}} \frac{\mathrm{d}}{\mathrm{d} t} \vec{r}+\frac{\partial H_{0}}{\partial \vec{p}} \frac{\mathrm{d}}{\mathrm{d} t} \vec{p}=0
$$

we obtain the dynamic equations called Hamilton equations,

$$
\begin{aligned}
& \frac{\mathrm{d}}{\mathrm{d} t} \vec{r}=\frac{\partial H_{0}}{\partial \vec{p}}=\frac{\partial}{\partial \vec{p}} T(\vec{p}) \\
& \frac{\mathrm{d}}{\mathrm{d} t} \vec{p}=-\frac{\partial H_{0}}{\partial \vec{r}}=-\frac{\partial}{\partial \vec{r}} U(\vec{r})-\underline{\text { Force. }}
\end{aligned}
$$

while the energy function of coordinates and momenta,

$$
H_{0}(\vec{r}, \vec{p})=\frac{\vec{p}^{2}}{2 M_{0}}+U(\vec{r})
$$

is called Hamiltonian. However, this classical description tells us nothing about the structure of the physical world. Only Quantum Mechanics tells us something about the structure of this world. Namely, that this world is composed of species of identical quantum particles. On one hand, experimentally, it has been found that these particles are of a wavy nature. On the other hand, one could find that the simplest way to define a quantum particle is by a wave packet, with momenta conjugated to coordinates and energy conjugated to time, with a single quantum constant $\hbar$ [9-11].

$$
\begin{aligned}
& \psi_{E}(\vec{r}, t)=\frac{1}{(2 \pi \hbar)^{3 / 2}} \int \varphi_{0}(\vec{p}, t) e^{\frac{i}{\hbar}(\vec{p} \vec{r}-E t)} d^{3} \vec{p} \\
& \varphi_{0}(\vec{p}, t)=\frac{1}{(2 \pi \hbar)^{3 / 2}} \int \psi_{E}(\vec{r}, t) e^{-\frac{i}{\hbar}(\vec{p} r-E t)} d^{3} \vec{r}
\end{aligned}
$$

In this case, one can define a momentum operator

$$
\vec{p}=-\mathrm{i} \hbar \frac{\partial}{\partial \vec{r}}
$$

and a Hamiltonian operator,

$$
H_{0}=\mathrm{i} \hbar \frac{\partial}{\partial t}=-\frac{\hbar^{2}}{2 M_{0}} \frac{\partial^{2}}{\partial \vec{r}^{2}}+U(\vec{r})=E
$$

With these operators one obtains eigenvalue equations, for momentum,

$$
-\mathrm{i} \hbar \frac{\partial}{\partial \vec{r}} \psi_{E}(\vec{r}, t)=\vec{p} \psi_{E}(\vec{r}, t)
$$

and for energy, as a Schrödinger equation:

$$
\left[-\frac{\hbar^{2}}{2 M_{0}} \frac{\partial^{2}}{\partial \vec{r}^{2}}+U(\vec{r})\right] \psi_{E}(\vec{r}, t)=E \psi_{E}(\vec{r}, t)
$$

However, when the group velocities are calculated for the wave packets (1.5), which with (1.1) are of the form 


$$
\begin{aligned}
& \psi_{E}(\vec{r}, t)=\frac{1}{(2 \pi \hbar)^{3 / 2}} \int \phi_{0}(\vec{p}, t) e^{\frac{\mathrm{i}}{\hbar}\{\vec{p} \vec{r}-[T(\vec{p})+U(\vec{r})] t\}} \mathrm{d}^{3} \vec{p} \\
& \phi_{0}(\vec{p}, t)=\frac{1}{(2 \pi \hbar)^{3 / 2}} \int \psi_{E}(\vec{r}, t) e^{-\frac{\mathrm{i}}{\hbar}\{\vec{p} \vec{r}-[T(\vec{p})+U(\vec{r})] t\}} \mathrm{d}^{3} \vec{r}
\end{aligned}
$$

we obtain an erroneous equation, contradictory to the corresponding Hamilton equation (1.3):

$$
\begin{aligned}
& \frac{d}{d t} \vec{r}=\frac{\partial H_{0}}{\partial \vec{p}}=\frac{\partial}{\partial \vec{p}} T(\vec{p})-O K \\
& \frac{d}{d t} \vec{p}=\frac{\partial H_{0}}{\partial \vec{r}}=\frac{\partial}{\partial \vec{r}} U(\vec{r})-\text { Erroneous equation }
\end{aligned}
$$

- a minus sign is missing [12-14]. We get back the minus sign only if instead the Hamiltonian $H_{0}(\vec{r}, \vec{p})=T(\vec{p})+U(\vec{r})$ we consider the Lagrangian

$$
L_{0}(\vec{r}, \dot{\vec{r}})=\vec{p} \dot{\vec{r}}-H_{0}(\vec{p}, \vec{r})=T(\vec{p})-U(\vec{r})=\frac{M \vec{v}^{2}}{2}-U(\vec{r})
$$

In his case, the wave packets (1.10) take a form

$$
\begin{aligned}
& \psi_{0}(\vec{r}, t)=\frac{1}{(2 \pi \hbar)^{3 / 2}} \int \phi_{0}(\vec{p}, t) e^{\frac{\mathrm{i}}{\hbar}\{\vec{p} r-[T(\vec{p})-U(\vec{r})] t\}} \mathrm{d}^{3} \vec{p} \\
& \phi_{0}(\vec{p}, t)=\frac{1}{(2 \pi \hbar)^{3 / 2}} \int \psi_{E}(\vec{r}, t) e^{-\frac{\mathrm{i}}{\hbar}\{\vec{p} \vec{r}-[T(\vec{p})-U(\vec{r})] t\}} \mathrm{d}^{3} \vec{r}
\end{aligned}
$$

with group velocities in agreement with the Hamilton equations (1.3):

$$
\begin{aligned}
& \frac{\mathrm{d}}{\mathrm{d} t} \vec{r}=\frac{\partial}{\partial \vec{p}} T(\vec{p})=\frac{\partial H_{0}}{\partial \vec{p}} \\
& \frac{\mathrm{d}}{\mathrm{d} t} \vec{p}=-\frac{\partial}{\partial \vec{r}} U(\vec{r})=-\frac{\partial H_{0}}{\partial \vec{r}}
\end{aligned}
$$

However, this description is still unrealistic, having an infinite spectrum of waves, as a function of the wave velocity $\vec{r}$. A finite spectrum is obtained when the relativistic Lagrangian $L_{0}(\dot{\vec{r}})$,

$$
L_{0}(\dot{\vec{r}}) \mathrm{d} t=-M_{0} c^{2} \sqrt{1-\frac{\dot{\vec{r}}^{2}}{c^{2}}} \mathrm{~d} t=-M_{0} c \mathrm{~d} s
$$

is considered in the time dependent phase of a particle wave:

$$
\begin{aligned}
& \Psi_{0}(\vec{r}, t)=\frac{1}{(2 \pi \hbar)^{3 / 2}} \int \varphi_{0}(\dot{\vec{r}}, t) e^{\frac{i}{\hbar}\left[M \dot{\vec{r}}-L_{0}(\vec{r}, \dot{\vec{r}}) t\right]} M^{3} d^{3} \dot{\vec{r}} \\
& \varphi_{0}(\dot{\vec{r}}, t)=\frac{1}{(2 \pi \hbar)^{3 / 2}} \int \psi(\vec{r}, t) e^{-\frac{i}{\hbar}\left[M \dot{\vec{r} r}-L_{0}(\vec{r}, \dot{\vec{r}}) t\right]} d^{3} \vec{r}
\end{aligned}
$$

From (1.15), we notice that the invariance of the spacetime interval of the Theory of Relativity is equivalent to the invariance of the time dependent phase variation of a quantum particle, and define a Relativistic Quantum Principle: The scalar tim-dependent phase variation of a quantum particle wave is an invariant for an arbitrary change of coordinates. On this basis, in section 2 we obtain the relativistic transform of the spacetime coordinates, and the relativistic dynamics of the particle waves. In section 3 , we consider a quantum particle in a field described by a scalar potential conjugated to time, and a vector potential conjugated to coordinates. We obtain the Lagrange equation as the velocity of the particle waves, the Lorentz force, and the Maxwell equations. In section 4, based on the Relativistic Quantum Principle, we obtain the dynamics of a quantum particle in electromagnetic field. In section 5, from the invariance of the time dependent phase of a quantum particle at an arbitrary change of coordinates, we obtain the relativistic transform of the electromagnetic field. In section 6, we obtain a relativistic equation for the quantum particle waves, show that the solution of the Schrödinger equation is only the amplitude of the wave function of a quantum particle, which also includes a rapidly varying factor depending on the particle rest mass, and derive the spin of a quantum particle wave. In section 7 , we consider the inversion of two particles as a double rotation of these particles, and obtain the spin-statistic relation. In section 8 , we consider a coordinate deformation according to the Theory of General Relativity, and obtain the dynamics of the particle waves in gravitational field. In section 9, we consider the particle waves as a distribution of matter described by a density and a velocity field, and find that these waves are perpendicular to the geodesic tracks. We derive an invariant for the matter density of a quantum particle, and an equation of conservation of this matter. Section 10 is for conclusions.

\section{Relativistic Kinematics and Dynamics}

A realistic particle has a finite spectrum as a function of the wave propagation velocity. A finite spectrum is obtained for a relativistic Lagrangian. According to (1.15), the invariance of the scalar time dependent phase variation $L_{0}(\dot{\vec{r}}) d t$ of a quantum particle wave function is equivalent to the invariance of the space-time interval $\mathrm{d} s$. For such a particle, we obtain the momentum,

$$
\vec{p}=\frac{\partial L_{0}}{\partial \dot{\vec{r}}}=\frac{M_{0} \dot{\vec{r}}}{\sqrt{1-\frac{\dot{\vec{r}}^{2}}{c^{2}}}}=M \dot{\vec{r}}
$$

and the mass,

$$
M=\frac{M_{0}}{\sqrt{1-\frac{\dot{\vec{r}}^{2}}{c^{2}}}}
$$

as functions of the particle velocity $\dot{\vec{r}}$ and the cut-off velocity $c$. The invariance of the space-time interval means that a change of coordinates is in fact a rotation of the space-time coordinates (Figure 1).

By a well-known calculation, the relativistic transform of the coordinate intervals is obtained for the quantum particle waves. Thus, from the coordinate transform,

$$
\begin{aligned}
& d x^{0}=d x^{0^{\prime}} \cos \phi+d x^{1^{\prime}} \sin \phi \\
& d x^{1}=d x^{1^{\prime}} \cos \phi-d x^{0^{\prime}} \sin \phi \\
& d x^{2}=d x^{2^{\prime}} \\
& d x^{3}=d x^{3^{\prime}}
\end{aligned}
$$

with the expressions of the rotation angle trigonometric functions, 


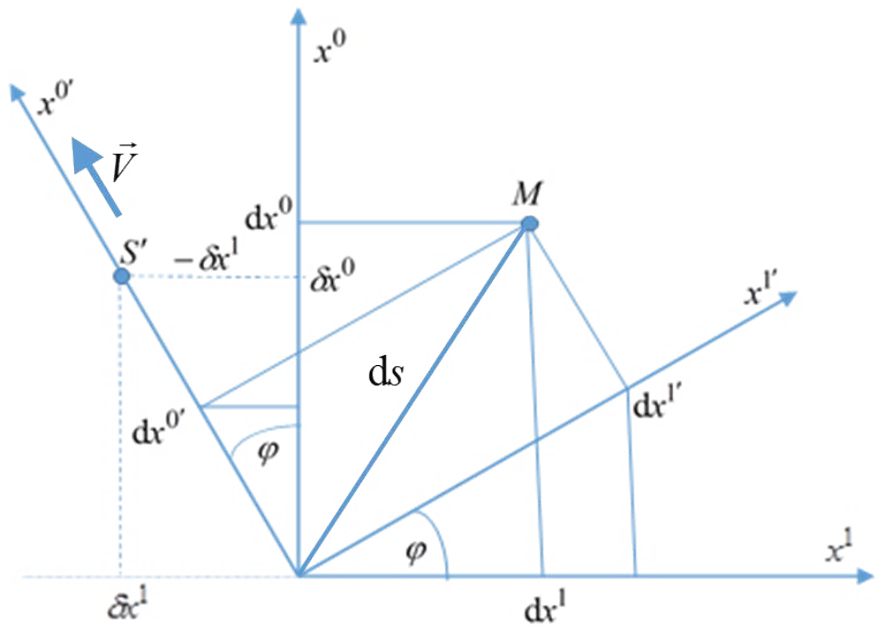

Figure 1. The rotation of the space-time system of coordinates for a velocity $\vec{V}$ of the system $\left(x^{0^{\prime}}=c t^{\prime}, x^{1^{\prime}}\right)$ over the system $\left(x^{0}=c t, x^{1}\right)$.

$$
\begin{aligned}
& \tan \varphi=\frac{-\delta x^{1}}{\delta x^{0}}=\frac{-\mathrm{i} \delta x}{c \delta t}=-\mathrm{i} \frac{\mathrm{V}}{\mathrm{c}} \\
& \cos \varphi=\frac{1}{\sqrt{1-\frac{V^{2}}{c^{2}}}} \\
& \sin \varphi=\frac{-\mathrm{i} \frac{\mathrm{V}}{\mathrm{c}}}{\sqrt{1-\frac{V^{2}}{c^{2}}}}
\end{aligned}
$$

for the coordinates of a quantum particle wave, we obtain the transform:

$$
\begin{aligned}
& d t=\frac{d t^{\prime}+\frac{V}{c^{2}} d x^{\prime}}{\sqrt{1-\frac{V^{2}}{c^{2}}}}, \quad d x=\frac{d x^{\prime}+V d t^{\prime}}{\sqrt{1-\frac{V^{2}}{c^{2}}}} \\
& d y=d y^{\prime}, \quad d z=d z^{\prime},
\end{aligned}
$$

which, in this case does not refer to the coordinates of some classical particle as in the conventional Theory of Relativity, but to the coordinates of the quantum particle waves.

\section{Electromagnetic Field}

When a particle with a charge $e$ is placed in a field of a vector potential $\vec{A}(\vec{r}, t)$ conjugated to the coordinates, and a scalar potential $U(\vec{r})$ conjugated to time, for the two wave packets of this particle

$$
\begin{aligned}
& \psi(\vec{r}, t)=\frac{1}{(2 \pi \hbar)^{3 / 2}} \int \varphi(\vec{P}, t) e^{\frac{i}{\hbar}[\vec{P} \vec{r}-L(\vec{r}, \dot{\vec{r}}, t) t]} d^{3} \vec{P} \\
& \varphi(\vec{P}, t)=\frac{1}{(2 \pi \hbar)^{3 / 2}} \int \psi(\vec{r}, t) e^{-\frac{i}{\hbar}[\vec{P} \vec{r}-L(\vec{r}, \vec{r}, t) t]} d^{3} \vec{r}
\end{aligned}
$$

a time dependent phase variation arises, with terms proportional to the coordinate variations, and the time variation,

$$
L(\vec{r}, \dot{\vec{r}}, t) d t=-M_{0} c^{2} \sqrt{1-\frac{\dot{\vec{r}}^{2}}{c^{2}}} d t+e \vec{A}(\vec{r}, t) d \vec{r}-e U(\vec{r}) d t
$$

In this case, we get a canonical momentum,

$$
\vec{P}=\frac{\partial}{\partial \dot{\vec{r}}} L(\vec{r}, \dot{\vec{r}}, t)=\frac{M_{0} \dot{\vec{r}}}{\sqrt{1-\frac{\dot{\vec{r}}^{2}}{c^{2}}}}+e \vec{A}(\vec{r}, t)=\vec{p}+e \vec{A}(\vec{r}, t)
$$

with a mechanical component depending on the particle mass and velocity, and an electromagnetic component as the product of the particle charge with the vector potential. For a quantum particle in a field we consider again the relativistic quantum principle: The time dependent phase variation of a quantum particle is the same in any system of coordinates.

We are in agreement with the Aharonov-Bohm effect [15]: the time-dependent phase of a quantum particle $\psi^{\prime}(\vec{r}, t)$,

$$
-\frac{\hbar^{2}}{2 m} \nabla^{2} \psi^{\prime}-V \psi^{\prime}=\mathrm{i} \hbar \frac{\partial \psi^{\prime}}{\partial t}
$$

in a magnetic field includes a term proportional to the space integral of the vector potential of this field:

$$
\begin{aligned}
& \psi(\vec{r}, t)=e^{i g(\vec{r})} \psi^{\prime}(\vec{r}, t) \\
& g(\vec{r})=\frac{e}{\hbar} \int_{0}^{\vec{r}} \vec{A}\left(\vec{r}^{\prime}\right) d \vec{r}^{\prime}
\end{aligned}
$$

This effect has been experimentally put out into evidence (Figure 2).

Besides this variation, we consider a time-dependent phase variation with a term proportional to the scalar potential and time, and the invariance of this phase. From equation (3.3), we obtain the electric force:

$$
\vec{F}_{e}=\frac{\mathrm{d}}{\mathrm{d} t} \vec{p}=\frac{\mathrm{d}}{\mathrm{d} t} \vec{P}-e \frac{\mathrm{d}}{\mathrm{d} t} \vec{A}(\vec{r}, t)
$$

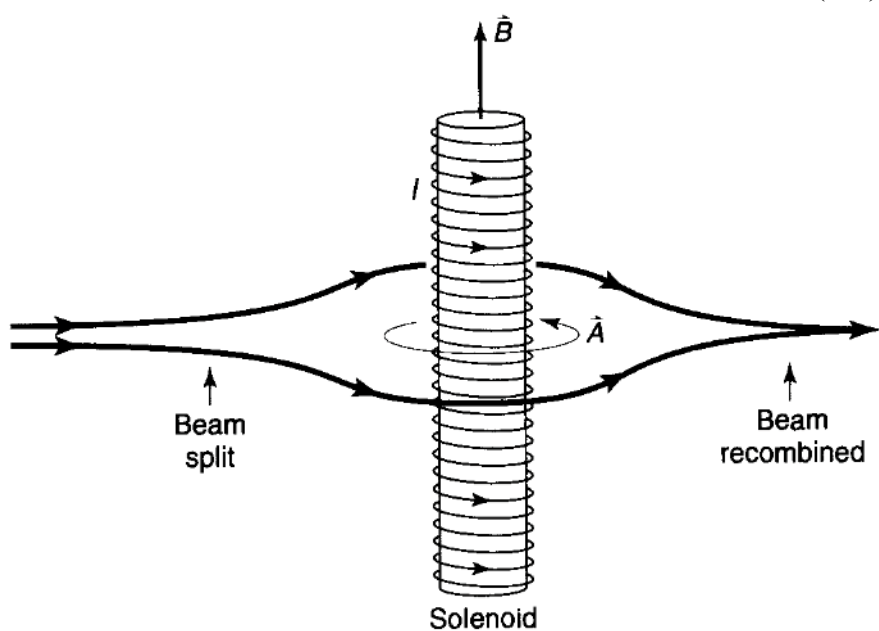

Figure 2. Experimental evidence of the Aharonov-Bohm effect: between the two electron beams, a phase difference arises due to the vector potential $\vec{A}[4]$. 
while from a particle wave velocity in the momentum space we obtain the Lagrange equation:

$$
\frac{\mathrm{d}}{\mathrm{d} t} \vec{P}=\frac{\partial}{\partial \vec{r}} L(\vec{r}, \dot{\vec{r}}, t)=e \frac{\partial}{\partial \vec{r}}[\vec{A}(\vec{r}, t) \dot{\vec{r}}]-e \frac{\partial}{\partial \vec{r}} U(\vec{r})
$$

With the vector formula:

$$
\dot{\vec{r}} \times\left[\frac{\partial}{\partial \vec{r}} \times A(\vec{r}, t)\right]=\frac{\partial}{\partial \vec{r}}[\dot{\vec{r}} A(\vec{r}, t)]-\left(\dot{\vec{r}} \frac{\partial}{\partial \vec{r}}\right) A(\vec{r}, t)
$$

we obtain the Lorentz force:

$$
\vec{F}_{e}=e \vec{E}(\vec{r}, t)+e \dot{\vec{r}} \times \vec{B}(\vec{r}, t)
$$

with the electric field,

$$
\vec{E}(\vec{r}, t)=-\frac{\partial}{\partial \vec{r}} U(\vec{r})-\frac{\partial}{\partial t} \vec{A}(\vec{r}, t)
$$

and the magnetic field,

$$
\vec{B}(\vec{r}, t)=\frac{\partial}{\partial \vec{r}} \times \vec{A}(\vec{r}, t)
$$

Taking into account that the curl of the gradient is null, from (3.8) with (3.9) we obtain the Faraday-Maxwell law of the electromagnetic induction:

$$
\frac{\partial}{\partial \vec{r}} \times \vec{E}(\vec{r}, t)=-\frac{\partial}{\partial t} \vec{B}(\vec{r}, t)
$$

Taking into account that the divergence of the curl is null, from (3.9) we obtain the Gauss-Maxwell law for the magnetic induction flow:

$$
\frac{\partial}{\partial \vec{r}} \vec{B}(\vec{r}, t)=0
$$

With the Gauge condition

$$
\frac{\partial}{\partial \vec{r}} \vec{A}(\vec{r}, t)=0
$$

we obtain the Gauss-Maxwell law for the electric field flow:

$$
\frac{\partial}{\partial \vec{r}} \vec{E}(\vec{r}, t)=-\frac{\partial^{2}}{\partial \vec{r}^{2}} U(\vec{r})=\frac{\rho(\vec{r})}{\varepsilon_{0}}
$$

with the Laplacian of the scalar potential as source of this divergence, considered as a ratio of the charge density $\rho(\vec{r})$ and the dimensional constant $\varepsilon_{0}$ called electric permittivity. Considering a current density under the action of the electric field,

$$
\vec{j}(\vec{r}, t)=\rho(\vec{r}, t) \dot{\vec{r}}=\sigma \vec{E}(\vec{r}, t) \Rightarrow \dot{\vec{r}} \times \vec{E}(\vec{r}, t)=0
$$

with the vector formula

$$
\begin{aligned}
\frac{\partial}{\partial \vec{r}} \times \underbrace{[\dot{\vec{r}} \times \vec{E}(\vec{r}, t)]}_{0} & =\dot{\vec{r}}\left[\frac{\partial}{\partial \vec{r}} \vec{E}(\vec{r}, t)\right]-\left(\dot{\vec{r}} \frac{\partial}{\partial \vec{r}}\right) \vec{E}(\vec{r}, t) \\
& =\dot{\vec{r}} \frac{\rho(\vec{r}, t)}{\varepsilon_{0}}-\left(\dot{\vec{r}} \frac{\partial}{\partial \vec{r}}\right) \vec{E}(\vec{r}, t),
\end{aligned}
$$

we obtain the time derivative of the electric field of the form:

$$
\varepsilon_{0} \frac{\mathrm{d}}{\mathrm{d} t} \vec{E}(\vec{r}, t)=\vec{j}(\vec{r}, t)+\varepsilon_{0} \frac{\partial}{\partial t} \vec{E}(\vec{r}, t)
$$

For the relation (3.10) of the two vector fields, asserting that a time variation of a magnetic field determines a curl of the electric field, we consider a symmetric relation, namely that a time variation of the electric field determines a curl of the magnetic field:

$$
\varepsilon_{0} \frac{\mathrm{d}}{\mathrm{d} t} \vec{E}(\vec{r}, t)=\frac{1}{\mu_{0}} \frac{\partial}{\partial \vec{r}} \times \vec{B}(\vec{r}, t)
$$

From (3.16) and (3.17), the Ampère-Maxwell law of the magnetic circuit is obtained. In this way we obtain the whole system of Maxwell equations:

$$
\begin{aligned}
& \frac{1}{\mu_{0}} \frac{\partial}{\partial \vec{r}} \times \vec{B}(\vec{r}, t)=\vec{j}(\vec{r})+\varepsilon_{0} \frac{\partial}{\partial t} \vec{E}(\vec{r}, t) \\
& \frac{\partial}{\partial \vec{r}} \times \vec{E}(\vec{r}, t)=-\frac{\partial}{\partial t} \vec{B}(\vec{r}, t) \\
& \frac{\partial}{\partial \vec{r}} \vec{B}(\vec{r}, t)=0 \\
& \frac{\partial}{\partial \vec{r}} \vec{E}(\vec{r}, t)=\frac{\rho(\vec{r}, t)}{\varepsilon_{0}}
\end{aligned}
$$

and the Lorentz force,

$$
\vec{F}_{e}=e \vec{E}(\vec{r}, t)+e \dot{\vec{r}} \times \vec{B}(\vec{r}, t)
$$

This force acts on the particle wave functions (3.1), with the time-dependent phase coefficient:

$$
L(\vec{r}, \dot{\vec{r}}, t)=-M_{0} c^{2} \sqrt{1-\frac{\dot{\vec{r}}^{2}}{c^{2}}}+e \vec{A}(\vec{r}, t) \dot{\vec{r}}-e U(\vec{r})
$$

as a function of the two potentials of the electromagnetic field:

$$
\begin{aligned}
& \vec{E}(\vec{r}, t)=-\frac{\partial}{\partial \vec{r}} U(\vec{r})-\frac{\partial}{\partial t} \vec{A}(\vec{r}, t) \\
& \vec{B}(\vec{r}, t)=\frac{\partial}{\partial \vec{r}} \times \vec{A}(\vec{r}, t) .
\end{aligned}
$$

It is interesting that by the hypothetical relation (3.17), we obtain a field propagating in waves with a velocity $1 / \sqrt{\varepsilon_{0} \mu_{0}}$, in agreement with the electromagnetic theory. For the physical consistency, this velocity takes the limit value of the velocity of the quantum particle waves (Figure 3):

$$
c=\frac{1}{\sqrt{\varepsilon_{0} \mu_{0}}}
$$

Otherwise, an electromagnetic field not interacting with a quantum particle, or a quantum particle not interacting with any electromagnetic field could exist, which is contradictory to our basic hypothesis of the particle-field interaction. 


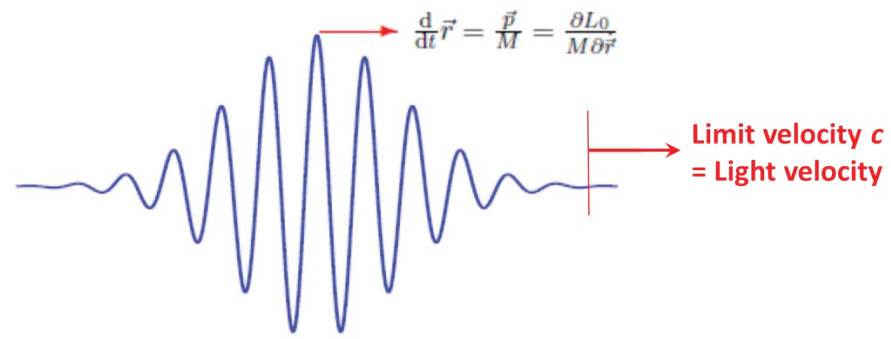

Figure 3. Wave packet of a quantum particle, with a limit velocity $c$ equal to the electromagnetic field velocity.

\section{DynamicsofaQuantumParticleinElectromagnetic Field}

We consider the space-time interval,

$\mathrm{d} s=c \sqrt{1-\frac{\dot{\vec{r}}^{2}}{c^{2}}} \mathrm{~d} t=\sqrt{-\mathrm{d} x_{i}^{2}}$

and the phase variation (3.2),

$d S=L(\vec{r}, \dot{\vec{r}}, t) d t=-M_{0} c d s+e \vec{A}(\vec{r}, t) d \vec{r}-e U(\vec{r}) d t$

as functions of the coordinate four-vector

$\left(x_{i}\right)=(x, y, z, i c t)$

and the field four-vector

$\left(A_{i}\right)=\left(A_{x}, A_{y}, A_{z}, \frac{\mathrm{i}}{c} U\right)$

For the wave velocity in the space of the momentum (3.3), from (3.1) we obtain a wave/group velocity of the form of the Lagrange equation:

$$
\frac{\mathrm{d}}{\mathrm{d} t} \frac{\partial}{\partial \dot{\vec{r}}} L(\vec{r}, \dot{\vec{r}}, t)=\frac{\partial}{\partial \vec{r}} L(\vec{r}, \dot{\vec{r}}, t)
$$

which means that the time-dependent phase of a quantum particle wave function is of the form of the action of the Lagrangian (3.20),

$$
S=\int L(\vec{r}, \dot{\vec{r}}, t) d t=\int\left(-M_{0} c d s+e A_{i} d x_{i}\right)
$$

In agreement with the principle of the least action,

$$
\delta S=\int\left(M_{0} c \frac{d x_{i}}{d s} \delta d x_{i}+e A_{i} \delta d x_{i}+e \delta A_{i} d x_{i}\right)=0
$$

for the velocity four-vector

$$
u_{i}=\frac{\mathrm{d} x_{i}}{\mathrm{~d} s}
$$

and the field four-tensor

$$
F_{i k}=\frac{\partial A_{k}}{\partial x_{i}}-\frac{\partial A_{i}}{\partial x_{k}}
$$

we obtain the dynamic equation

$$
M_{0} c \frac{d u_{i}}{d s}=e F_{i k} u_{k}
$$

or

$$
\frac{d}{d t}\left(M \frac{d x_{i}}{d t}\right)=e F_{i k} \frac{d x_{k}}{d t}
$$

with the relativistic mass

$M=\gamma M_{0}$

which depends on the relativistic coefficient

$$
\gamma=\frac{1}{\sqrt{1-\frac{V^{2}}{c^{2}}}}
$$

With the explicit expression of the field four-tensor

$$
\left(F_{i k}\right)=\left(\begin{array}{cccc}
0 & B_{z} & -B_{y} & -\frac{i}{c} E_{x} \\
-B_{z} & 0 & B_{x} & -\frac{i}{c} E_{y} \\
B_{y} & -B_{x} & 0 & -\frac{i}{c} E_{z} \\
\frac{i}{c} E_{x} & \frac{i}{c} E_{y} & \frac{i}{c} E_{z} & 0
\end{array}\right)
$$

we obtain the Lorentz force,

$$
\frac{\mathrm{d}}{\mathrm{d} t}(M \dot{\vec{r}})=e \vec{E}+e \dot{\vec{r}} \times \vec{B}
$$
field.

for the interaction of a particle wave with an electromagnetic

\section{Relativistic Transform of a Field Interacting with a Quantum Particle}

For a coordinate four-vector transform,

$$
x_{i}=\alpha_{i j} x_{j}^{\prime}, x_{j}^{\prime}=\alpha_{j i}^{-1} x_{i}
$$

with the transformation matrix,

$$
\left(\alpha_{i j}\right)=\left(\begin{array}{cccc}
\gamma & 0 & 0 & -i \gamma \frac{V}{c} \\
0 & 1 & 0 & 0 \\
0 & 0 & 1 & 0 \\
i \gamma \frac{V}{c} & 0 & 0 & 1
\end{array}\right)
$$

in the least action equation (4.7), we obtain an invariant including a mechanical term and an electromagnetic term:

$$
\delta S=\int[\underbrace{-M_{0} c \mathrm{~d} u_{i} \delta x_{i}}_{\text {mechanical }}+\underbrace{F_{k} u_{k} \delta x_{i} \mathrm{~d} s}_{\text {electromagnetic }}]=0
$$

Since the mechanical term is invariant to any change of coordinates, 
$d u_{i} \delta x_{i}=\alpha_{i j} \alpha_{i k} d u_{j}^{\prime} \delta x_{k}^{\prime}=\alpha_{j i}^{-1} \alpha_{i k} d u_{j}^{\prime} \delta x_{k}^{\prime}=\delta_{j k} d u_{j}^{\prime} \delta x_{k}^{\prime}=d u_{j}^{\prime} \delta x_{j}^{\prime}$

the electromagnetic term must be also an invariant,

$$
F_{i k} u_{k} \delta x_{i}=F_{i k} \alpha_{k l} u_{l}^{\prime} \alpha_{i j} \delta x_{j}^{\prime}=F_{j l}^{\prime} u_{l}^{\prime} \delta x_{j}^{\prime}
$$

or

$$
F_{j l}^{\prime} u_{l}^{\prime} \delta x_{j}^{\prime}=F_{j l}^{\prime} \alpha_{l k}^{-1} u_{k} \alpha_{j i}^{-1} \delta x_{i}=F_{j l}^{\prime} \alpha_{k l} \underline{u_{k}} \underline{\alpha_{i j}} \underline{\underline{\delta x_{i}}}=F_{i k} \underline{\underline{u_{k}}} \underline{\underline{\delta x_{i}}}
$$

On this basis, we find the transform for a change of coordinates of a field interacting with a quantum particle

$$
F_{j l}^{\prime}=F_{i k} \alpha_{i j} \alpha_{k l}
$$

$$
\stackrel{\text { or }}{F_{i k}}=\alpha_{i j} \alpha_{k l} F_{j l}^{\prime}
$$

with the explicit form:

$$
\begin{aligned}
& E_{x}=E_{x^{\prime}}, \quad E_{y}=\frac{E_{y^{\prime}}+V B_{z^{\prime}}}{\sqrt{1-\frac{V^{2}}{c^{2}}}}, \quad E_{z}=\frac{E_{z^{\prime}}-V B_{y^{\prime}}}{\sqrt{1-\frac{V^{2}}{c^{2}}}}, \\
& B_{z}=B_{z^{\prime}}, \quad B_{y}=\frac{B_{y^{\prime}}-\frac{V}{c^{2}} E_{x^{\prime}}}{\sqrt{1-\frac{V^{2}}{c^{2}}}}, \quad B_{z}=\frac{B_{z^{\prime}}+\frac{V}{c^{2}} E_{y^{\prime}}}{\sqrt{1-\frac{V^{2}}{c^{2}}}} .
\end{aligned}
$$

\section{Particle Wave Function and Spin}

From the Lagrange equation obtained as the group velocity (3.5) with the momentum expression (3.3),

$$
\frac{\mathrm{d}}{\mathrm{d} t} \vec{P}=\frac{\mathrm{d}}{\mathrm{d} t} \frac{\partial}{\partial \dot{\vec{r}}} L(\vec{r}, \dot{\vec{r}}, t)=\frac{\partial}{\partial \vec{r}} L(\vec{r}, \dot{\vec{r}}, t)
$$

and the Hamiltonian definition

$$
H(\vec{P}, \vec{r}, t)=\vec{P} \dot{\vec{r}}-L(\vec{r}, \dot{\vec{r}}, t)
$$

as a conservative function,

$$
\begin{aligned}
& \mathrm{d} H(\vec{P}, \vec{r}, t) \equiv \frac{\partial}{\partial \vec{P}} H(\vec{P}, \vec{r}, t) \mathrm{d} \vec{P}+\frac{\partial}{\partial \vec{r}} H(\vec{P}, \vec{r}, t) \mathrm{d} \vec{r}+\frac{\partial H}{\partial t} \mathrm{~d} t
\end{aligned}
$$

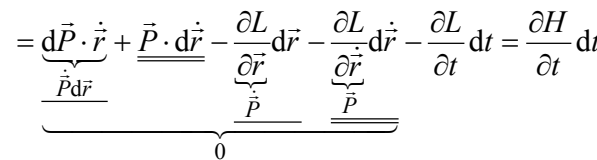

we obtain the Hamilton equation

$$
\begin{aligned}
& \dot{\vec{r}}=\frac{\partial}{\partial \vec{P}} H(\vec{P}, \vec{r}, t) \\
& \dot{\vec{P}}=-\frac{\partial}{\partial \vec{r}} H(\vec{P}, \vec{r}, t) \\
& \frac{\partial}{\partial t} H(\vec{P}, \vec{r}, t)=-\frac{\partial}{\partial t} L(\vec{r}, \dot{\vec{r}}, t)
\end{aligned}
$$

At the same time, with the definition expression (6.2), the wave packets (3.1) take a form

$$
\begin{aligned}
& \psi(\vec{r}, t)=\frac{1}{(2 \pi h)^{3 / 2}} \int \phi(\vec{P}, t) e^{\frac{\mathrm{i}}{\hbar}\{\vec{P} \vec{r}-[\vec{P} \dot{\vec{r}}-H(\vec{P}, \vec{r})] t} \mathrm{d}^{3} \vec{P} \\
& \phi(\vec{P}, t)=\frac{1}{(2 \pi h)^{3 / 2} \int \psi(\vec{r}, t) e^{-\frac{\mathrm{i}}{\hbar}\{\vec{P} \vec{r}-[\vec{P} \dot{\vec{r}}-H(\vec{P}, \vec{r})] t\}} \mathrm{d}^{3} \vec{r}}
\end{aligned}
$$

as solutions of a wave equation

$i \hbar \frac{\partial}{\partial t} \psi(\vec{r}, t)=-i \hbar \dot{\vec{r}} \frac{\partial}{\partial \vec{r}} \psi(\vec{r}, t)-H(\vec{P}, \vec{r}) \psi(\vec{r}, t)$

which, compared to the Schrödinger equation includes a term depending on the velocity.

On the other hand, from (3.1) we notice that, in a classical approximation,

$$
\begin{gathered}
L(\vec{r}, \dot{\vec{r}}, t)=-M_{0} c^{2} \sqrt{1-\frac{\dot{\vec{r}}^{2}}{c^{2}}}+e \vec{A}(\vec{r}, t) \dot{\vec{r}}-e U(\vec{r}) \\
\approx-M_{0} c^{2}+\frac{M_{\vec{r}}^{2}}{2}+e \vec{A}(\vec{r}, t) \dot{\vec{r}}-e U(\vec{r})
\end{gathered}
$$

the Schrödinger wave function $\psi_{E}(\vec{r}, t)$, we usually use in our studies, in fact is not the particle wave function, bat only an amplitude of a wave function including a rapidly varying factor,

$$
\psi(\vec{r}, t)=e^{\frac{i}{\hbar}\left[M_{0} c^{2}+2 e U(\vec{r})\right] t} \psi_{E}(\vec{r}, t)
$$

We consider an electromagnetic field with a constant scalar potential for a stationary electric field, and a time-dependent vector potential for a coherent radiation field. From equation (6.2) with (3.3) and (3.20), we notice that although the vector potential $\vec{A}(\vec{r}, t)$ depends on time, the Hamiltonian does not:

$$
\begin{aligned}
& H(\vec{P}, \vec{r}, t)=\vec{P} \dot{\vec{r}}-L(\vec{r}, \dot{\vec{r}}, t) \\
& =\frac{M_{0} \dot{\vec{r}}^{2}}{\sqrt{1-\frac{\dot{\vec{r}}^{2}}{c^{2}}}}+e \vec{A}(\vec{r}, t) \dot{\vec{r}}-\left(-M_{0} c^{2} \sqrt{1-\frac{\dot{\vec{r}}^{2}}{c^{2}}}+e \vec{A}(\vec{r}, t) \dot{\vec{r}}-e U(\vec{r})\right)
\end{aligned}
$$

With this Hamiltonian we, obtain the total energy as a function of the mechanical energy and the potential energy in electric field:

$$
H(\vec{P}, \vec{r}, t)=\frac{M_{0} c^{2}}{\sqrt{1-\frac{\dot{\vec{r}}^{2}}{c^{2}}}}+e U(\vec{r})=E(\vec{r}, \dot{\vec{r}})
$$

With the mechanical energy as a function of the canonical momentum (3.3),

$$
\frac{M_{0}^{2} c^{2}}{1-\frac{\dot{\vec{r}}^{2}}{c^{2}}}=\frac{M_{0}^{2} \dot{\vec{r}}^{2}}{1-\frac{\dot{\vec{r}}^{2}}{c^{2}}}+M_{0}^{2} c^{2}=[\vec{P}-e \vec{A}(\vec{r}, t)]^{2}+M_{0}^{2} c^{2}
$$

we obtain the canonical form of the Hamiltonian

$$
H(\vec{P}, \vec{r}, t)=c \sqrt{M_{0}^{2} c^{2}+[\vec{P}-e \vec{A}(\vec{r}, t)]^{2}}+e U(\vec{r})
$$

According to Dirac's well-known spin theory, we consider 
the Schrödinger equation

$\mathrm{i} \hbar \frac{\partial}{\partial t} \psi_{E}(\vec{r}, t)=H \psi_{E}(\vec{r}, t)$

with the Hamiltonian

$$
\begin{aligned}
& H(\vec{p}, \vec{r}, t)=c \sqrt{\vec{p}^{2}+M_{0}^{2} c^{2}}+e U(\vec{r})=c \\
& \left(\alpha_{1} p_{1}+\alpha_{2} p_{2}+\alpha_{3} p_{3}+\alpha_{4} M_{0} c\right)+e U(\vec{r})
\end{aligned}
$$

which depends on Dirac's spin operators

$\alpha_{0}=\left(\begin{array}{cc}\hat{1} & 0 \\ 0 & -\hat{1}\end{array}\right), \alpha_{1}=\left(\begin{array}{cc}0 & \sigma_{1} \\ \sigma_{1} & 0\end{array}\right), \alpha_{2}=\left(\begin{array}{cc}0 & \sigma_{2} \\ \sigma_{2} & 0\end{array}\right), \alpha_{3}=\left(\begin{array}{cc}0 & \sigma_{3} \\ \sigma_{3} & 0\end{array}\right)$

as functions of Pauli's spin operators,

$\sigma_{1}=\left(\begin{array}{ll}0 & 1 \\ 1 & 0\end{array}\right), \sigma_{2}=\left(\begin{array}{cc}0 & -\mathrm{i} \\ \mathrm{i} & 0\end{array}\right), \sigma_{3}=\left(\begin{array}{cc}1 & 0 \\ 0 & -1\end{array}\right)$

These operators satisfy the commutation relations:

$$
\begin{aligned}
& \left\{\alpha_{i}, \alpha_{j}\right\}=\alpha_{i} \alpha_{j}+\alpha_{j} \alpha_{i}=2 \delta_{i j} \\
& \left\{\sigma_{i}, \sigma_{j}\right\}=\sigma_{i} \sigma_{j}+\sigma_{j} \sigma_{i}=2 \delta_{i j}
\end{aligned}
$$

With the notation

$$
\vec{\alpha}=\left(\alpha_{1}, \alpha_{2}, \alpha_{3}\right)
$$

the Schrödinger equation (6.13) with the Hamiltonian (6.14) takes a form:

$$
\left[c\left(\alpha_{0} M_{0} c+\vec{\alpha} \vec{p}\right)+e U(\vec{r})\right] \psi_{E}(\vec{r}, t)=E \psi_{E}(\vec{r}, t)
$$

with a wave function which can be written as a vector with two components, or a vector with four components,

$$
\psi_{E}=\left(\begin{array}{l}
\psi_{1} \\
\psi_{2}
\end{array}\right)=\left(\begin{array}{l}
\phi_{1} \\
\phi_{2} \\
\phi_{3} \\
\phi_{4}
\end{array}\right)
$$

We obtain the two-dimensional Schrödinger-Dirac equation,

$$
c\left[\left(\begin{array}{c}
\psi_{1}(\vec{r}) \\
-\psi_{2}(\vec{r})
\end{array}\right) M_{0} c+\left(\begin{array}{l}
\vec{\sigma} \psi_{2}(\vec{r}) \\
\vec{\sigma} \psi_{1}(\vec{r})
\end{array}\right) \vec{p}\right]+e U(\vec{r})\left(\begin{array}{c}
\psi_{1}(\vec{r}) \\
\psi_{2}(\vec{r})
\end{array}\right)=E\left(\begin{array}{l}
\psi_{1}(\vec{r}) \\
\psi_{2}(\vec{r})
\end{array}\right)
$$

with two coupled Schrödinger-Dirac equations for the two components $\psi_{1}(\vec{r})$ and $\psi_{2}(\vec{r})$,

$$
\begin{aligned}
& {\left[M_{0} c^{2}+e U(\vec{r})\right] \psi_{1}(\vec{r})+c \vec{\sigma} \vec{p} \psi_{2}(\vec{r})=E \psi_{1}(\vec{r})} \\
& {\left[-M_{0} c^{2}+e U(\vec{r})\right] \psi_{2}(\vec{r})+c \vec{\sigma} \vec{p} \psi_{1}(\vec{r})=E \psi_{2}(\vec{r})}
\end{aligned}
$$

where

$$
\vec{\sigma}=\left(\sigma_{1}, \sigma_{2}, \sigma_{3}\right)
$$

By eliminating the coupling terms, the two SchrödingerDirac equations take non-linear forms, which, in a nonrelativistic approximation, small velocity, small electric potential, become linear:

$$
\begin{aligned}
& \underbrace{\left[E+M_{0} c^{2}-e U(\vec{r})\right]}_{\approx 2 M_{0} c^{2}}[\underbrace{E-M_{0} c^{2}}_{E_{c}}-e U(\vec{r})] \psi_{1}(\vec{r})=c^{2}(\vec{\sigma} \vec{p})^{2} \psi_{1}(\vec{r}) \\
& {[\underbrace{E-M_{0} c^{2}}_{\approx E_{c}}-e U(\vec{r})]\left[E+M_{0} c^{2}-e U(\vec{r})\right] \psi_{2}(\vec{r})=c^{2}(\vec{\sigma} \vec{p})^{2} \psi_{2}(\vec{r})}
\end{aligned}
$$

In the non-relativistic approximation, we get SchrödingerDirac equations

$$
\begin{aligned}
& {\left[\frac{(\vec{\sigma} \vec{p})^{2}}{2 M_{0}}+e U(\vec{r})\right] \psi_{1}(\vec{r})=E_{c} \psi_{1}(\vec{r})} \\
& {\left[\frac{(\vec{\sigma} \vec{p})^{2}}{2 M_{0}}+e U(\vec{r})\right] \psi_{2}(\vec{r})=E_{c} \psi_{2}(\vec{r})}
\end{aligned}
$$

for the classical energy

$$
E_{c}=E-M_{0} c^{2}
$$

The Hamiltonian of these equations includes a kinetic term depending on the momentum and the Pauli spin operators,

$(\overrightarrow{\sigma p})^{2}=\left(\sigma_{1} p_{1}+\sigma_{2} p_{2}+\sigma_{3} p_{3}\right)^{2}=\vec{p}^{2}+\mathrm{i} \vec{\sigma}(\vec{p} \times \vec{p})$

With the mechanical momentum in a magnetic field,

$$
\vec{p}=\vec{P}-e \vec{A}(\vec{r}, t)=-\mathrm{i} \hbar \nabla-e \vec{A}(\vec{r}, t)
$$

we obtain the vector product of equation (6.28) of the form

$$
\begin{aligned}
& \vec{p} \times \vec{p}=(-\mathrm{i} \hbar \nabla-e \vec{A}) \times(-\mathrm{i} \hbar \nabla-e \vec{A})=\mathrm{i} \hbar e(\nabla \times \vec{A}+\vec{A} \times \nabla)=\mathrm{i} e \hbar \vec{B} \\
& (\nabla \times \vec{A}+\vec{A} \times \nabla) \phi=\nabla \times(\vec{A} \phi)+\vec{A} \times(\nabla \phi)=\phi \overbrace{(\nabla \times \vec{A}}^{\hat{A}})
\end{aligned}
$$

Thus, in the kinetic term of the Hamiltonian, besides the mechanical term, proportional to the square of the mechanical momentum, we obtain a magnetic potential, proportional to the magnetic field, as of a proper rotation, called spin:

$$
(\overrightarrow{\sigma p})^{2}=\vec{p}^{2}-e \hbar \vec{\sigma} \vec{B}
$$

We obtain Schrödinger-Dirac equations with a Hamiltonian including the kinetic energy, the electric potential energy, and a potential energy in magnetic field, due to the particle spin:

$$
\begin{aligned}
& {\left[\frac{\vec{p}^{2}}{2 M_{0}}-\vec{\mu}_{s} \vec{B}+e U(\vec{r})\right] \psi_{1}(\vec{r})=E_{c} \psi_{1}(\vec{r})} \\
& {\left[\frac{\vec{p}^{2}}{2 M_{0}}-\vec{\mu}_{s} \vec{B}+e U(\vec{r})\right] \psi_{2}(\vec{r})=E_{c} \psi_{2}(\vec{r})}
\end{aligned}
$$

with the spin magnetic moment

$$
\vec{\mu}_{s}=\frac{\hbar e}{2 M_{0}} \vec{\sigma}
$$

and the component

$$
\mu_{3}=\frac{\hbar e}{2 M_{0}} \sigma_{3}
$$

in the direction of the magnetic field. Taking into account the commutation relation

$$
\left[H, j_{3}\right]=\left[H, l_{3}+s_{3}\right]=0
$$


of the Hamiltonian

$$
H=c\left(\alpha_{1} p_{1}+\alpha_{2} p_{2}+\alpha_{3} p_{3}+\alpha_{4} M_{0} c\right)
$$

with the total angular momentum, which includes the orbital angular momentum $l_{3}$ and the spin angular momentum $s 3$, we obtain a relation between the spin angular momentum and the orbital angular momentum which is known:

$$
\left[H, s_{3}\right]=-\left[H, l_{3}\right]
$$

With the commutation relations

$$
\left\lfloor p_{i}, l_{j}\right\rfloor=\mathrm{i} \hbar \delta_{\mathrm{ijk}} p_{k}
$$

from (6.35) and (6.36) we obtain the commutation relations

$$
\begin{aligned}
& {\left[\alpha_{1}, s_{3}\right]=-i \hbar \alpha_{2}} \\
& {\left[\alpha_{2}, s_{3}\right]=\underline{i \hbar \alpha_{1}}} \\
& {\left[\alpha_{3}, s_{3}\right]=0} \\
& {\left[\alpha_{4}, s_{3}\right]=0}
\end{aligned}
$$

These equations have a solution of the form

$$
s_{3}=s \alpha_{1} \alpha_{2}
$$

with the coefficient

$$
s=-\mathrm{i} \frac{\hbar}{2}
$$

Thus, we obtain the spin angular momentum in the direction of the magnetic field

$$
s_{3}=-\mathrm{i} \frac{\hbar}{2} \alpha_{1} \alpha_{2}=\frac{\hbar}{2}\left(\begin{array}{cc}
\sigma_{3} & 0 \\
0 & \sigma_{3}
\end{array}\right)
$$

with the Eigen value equations

$$
\begin{aligned}
& s_{3} \psi_{1}=\frac{\hbar}{2} \sigma_{3} \psi_{1} \\
& s_{3} \psi_{2}=\frac{\hbar}{2} \sigma_{3} \psi_{2}
\end{aligned}
$$

From (6.34) and (6.41) we obtain the gyromagnetic ratio

$$
g_{s}=\frac{\mu_{3}}{s_{3}}=\frac{e}{M_{0}}
$$

In this way, the spin is obtained from the relativistic quantum principle, in the framework of a unitary relativistic quantum theory.

\section{Spin-Statistic Relation}

We consider a system of two particles, in the states $\left|i_{1}\right\rangle$ and $\left|i_{2}\right\rangle$, with the position vectors $\vec{r}_{1}$ and $\vec{r}_{2}$ (Figure 4).

For a two particle wave function

$$
\left\langle\vec{r}_{1}, \vec{r}_{2} \mid i_{1}, i_{2}\right\rangle
$$

we define an inversion operator $I$ :

$$
\left\langle\vec{r}_{2}, \vec{r}_{1} \mid i_{1}, i_{2}\right\rangle=I\left\langle\vec{r}_{1}, \vec{r}_{2} \mid i_{1}, i_{2}\right\rangle
$$

By applying two times this operator,

$$
\left\langle\vec{r}_{1}, \vec{r}_{2} \mid i_{1}, i_{2}\right\rangle=I\left\langle\vec{r}_{2}, \vec{r}_{1} \mid i_{1}, i_{2}\right\rangle=I^{2}\left\langle\vec{r}_{1}, \vec{r}_{2} \mid i_{1}, i_{2}\right\rangle
$$

we find the Eigen values

$$
I^{2}=1 \begin{cases}I_{1}=-1 & \text { for Fermions } \\ I_{2}=1 & \text { for Bosons }\end{cases}
$$

On the other hand, we notice that an inversion is equivalent with a double rotation with the angle $\pi$ (Figure 4),

$$
I=R_{\pi}^{(1)} R_{\pi}^{(2)}
$$

For a wave function rotation with a differential angle $\delta \vec{\alpha}$,

$$
\begin{aligned}
\psi(\vec{r}+\delta \vec{\alpha} \times \vec{r}) & =\psi(\vec{r})+\delta \vec{\alpha} \times \vec{r} \frac{\partial}{\partial \vec{r}} \psi(\vec{r}) \\
& =\psi(\vec{r})+\delta \vec{\alpha} \cdot \underbrace{\frac{\partial}{\partial \vec{r}}}_{\mathrm{i} \vec{r}=\mathrm{i} \vec{S}} \psi(\vec{r}) \\
& =e^{\mathrm{i} \vec{S} \delta \vec{\alpha}} \psi(\vec{r})
\end{aligned}
$$
$\vec{S}$

$R_{\delta \vec{\alpha}}=e^{i \vec{S} \delta \vec{\alpha}}$

which, for a rotation with an arbitrary angle $\vec{\alpha}$ generates $R_{\vec{\alpha}}=e^{i S \vec{\alpha}}$

For $\alpha=\pi$ we obtain

$$
R_{\pi}^{(1)}=R_{\pi}^{(2)}=e^{\mathrm{i} \pi S}
$$

With this expression, from (7.4) and (7.5) we obtain the inversion eigenvalues as a function of spin,

$$
I=e^{i 2 \pi S}\left\{\begin{array}{lll}
I_{1}=e^{i 2 \pi S_{1}}=-1 & \Rightarrow S_{1}=\frac{1}{2} & \text { - Fermions } \\
I_{2}=e^{i 2 \pi S_{2}}=1 \Rightarrow S_{2}=1 & \text { - Bosons }
\end{array}\right.
$$

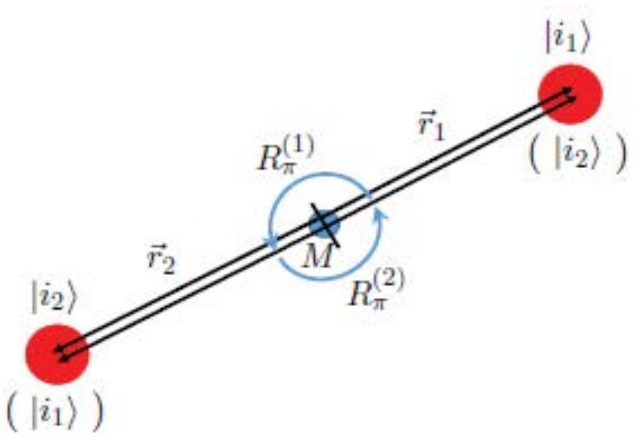

Figure 4. Two-particle system - a particle inversion equivalent to a double rotation with the angle $\pi$. 


\section{Quantum Particle in Gravitational Field}

We consider the wave functions (1.16) in a system of curvilinear coordinates $\left(x^{\alpha}\right)=\left(x^{0}, x^{i}\right), x^{0}=c t$,

$$
\begin{aligned}
& \psi\left(x^{i}, t\right)=\frac{1}{(2 \pi \hbar)^{3 / 2}} \int \varphi\left(\dot{x}^{i}, t\right) e^{\frac{i}{\hbar} M_{0} c \int\left(-g_{i j} \dot{x}^{i} \dot{x}^{j}+1\right) d s} M_{0}^{3} c^{3} \frac{\partial(\dot{x}, \dot{y}, \dot{z})}{\partial\left(\dot{x}^{1}, \dot{x}^{2}, \dot{x}^{3}\right)} d \dot{x}^{1} d \dot{x}^{2} d \dot{x}^{3} \\
& \varphi\left(\dot{x}^{i}, t\right)=\frac{1}{(2 \pi \hbar)^{3 / 2}} \int \psi\left(x^{i}, t\right) e^{-\frac{i}{\hbar} M_{0} \int\left(-g_{i} \dot{x}^{i} \dot{x}^{j}+1\right) d s} \frac{\partial(x, y, z)}{\partial\left(x^{1}, x^{2}, x^{3}\right)} d x^{1} d x^{2} d x^{3}
\end{aligned}
$$

with the time-space differential interval ds,

$$
d s^{2}=g_{\alpha \beta} d x^{\alpha} d x^{\beta}
$$

and use Dirac's formalism of General Theory of Relativity [16]. In (8.1), we used the notation:

$$
\dot{x}^{i}=\frac{d x^{i}}{d s}
$$

From (8.2), we notice that

$$
g_{\alpha \beta} \dot{x}^{\alpha} \dot{x}^{\beta}=g_{\alpha \beta} \frac{d x^{\alpha}}{d s} \frac{d x^{\beta}}{d s}=1
$$

For a non-relativistic case, small particles compared with the non-uniformities of any gravitational field, small velocities, we consider:

$$
d s=\sqrt{g_{00} d x^{02}+\underbrace{g_{i j} d x^{i} d s^{j}}_{\text {negligible }}} \approx d x^{0}=c d t \Rightarrow \frac{d s}{d t} \approx \frac{d x^{0}}{d t}=c
$$

and linearize the wave phases:

$$
\begin{aligned}
\int\left(-g_{i j} \dot{x}^{i} \dot{x}^{j} d s+d s\right) & =\int\left(-g_{i j} \dot{x}^{i} d x^{j}+\sqrt{c^{2} d t^{2}+g_{i j} d x^{i} d x^{j}}\right) \\
= & \int\left(-g_{i j} \dot{x}^{i} d x^{j}+c d t \sqrt{1+g_{i j} \dot{x}^{i} \dot{x}^{j}}\right) \\
= & \int\left[-g_{i j} \dot{x}^{i} d x^{j}+c d t\left(1+\frac{1}{2} g_{i j} \dot{x}^{i} \dot{x}^{j}\right)\right] \\
= & \int\left(-\frac{1}{2} g_{i j} \dot{x}^{i} d x^{j}+c \sqrt{g_{\alpha \beta} \dot{x}^{\alpha} \dot{x}^{\beta}} d t\right) \\
= & -\frac{1}{2} g_{i j} \dot{x}^{i} x^{j}+c \sqrt{g_{\alpha \beta} \dot{x}^{\alpha} \dot{x}^{\beta}} t
\end{aligned}
$$

In this way, for a quantum particle we obtain wave packets with linear phases in space and time,

$$
\begin{aligned}
& \psi\left(x^{i}, t\right)=\frac{1}{(2 \pi \hbar)^{3 / 2}} \int \varphi\left(\dot{x}^{i}, t\right) e^{\frac{i}{h^{h}} M_{0} c\left(-\frac{1}{2} g_{i j} \dot{y}^{i} x^{j}+c \sqrt{g_{\alpha \beta} \dot{x}^{x^{*} \dot{x}^{3}}} t\right)} M_{0}^{3} c^{3} \frac{\partial(\dot{x}, \dot{y}, \dot{z})}{\partial\left(\dot{x}^{1}, \dot{x}^{2}, \dot{x}^{3}\right)} d \dot{x}^{1} d \dot{x}^{2} d \dot{x}^{3} \\
& \varphi\left(\dot{x}^{i}, t\right)=\frac{1}{(2 \pi \hbar)^{3 / 2}} \int \psi\left(x^{i}, t\right) e^{-\frac{i}{\hbar} M_{0}\left(-\frac{1}{2} g_{i j} \dot{x}^{i} x^{j}+c \sqrt{g_{\alpha \beta} \dot{x}^{\alpha} \dot{x}^{\beta}} t\right)} \frac{\partial(x, y, z)}{\partial\left(x^{1}, x^{2}, x^{3}\right)} d x^{1} d x^{2} d x^{3}
\end{aligned}
$$

For this wave packet we obtain a self-consistent expression of the wave velocity,

$$
v^{j}=\frac{d x^{j}}{d t}=\frac{\partial}{\frac{1}{2} \partial\left(g_{i j} \dot{x}^{i}\right)}\left(c \sqrt{g_{\alpha \beta} \dot{x}^{\alpha} \dot{x}^{\beta}}\right)=2 c \frac{\dot{x}_{j}}{2 \underbrace{\sqrt{g_{\alpha \beta} \dot{x}^{\alpha} \dot{x}^{\beta}}}_{1}}=c \frac{d x^{j}}{d s}
$$

and the acceleration of a particle on a geodesic as a function of the Christoffel symbol $\Gamma_{\mu \nu}^{j}$ of the second kind,

$$
a^{j}=\frac{d}{d t} v^{j}=c^{2} \frac{d^{2} x^{j}}{d s^{2}}=-c^{2} \Gamma_{\mu \nu}^{j} \frac{d x^{\mu}}{d s} \frac{d x^{\nu}}{d s}
$$

We introduce the Christoffel symbol of the first kind, and consider the expression of this symbol as a function of the metric tensor derivatives:

$$
\begin{aligned}
& a^{j}=-c^{2} \Gamma_{\mu \nu}^{j} \frac{d x^{\mu}}{d s} \frac{d x^{v}}{d s}=-c^{2} g^{j \lambda} \Gamma_{\lambda \mu \nu} \frac{d x^{\mu}}{d s} \frac{d x^{\nu}}{d s}=- \\
& c^{2} g^{j \lambda} \frac{1}{2}\left(g_{\lambda \mu, v}+g_{\lambda v, \mu}-g_{\mu v, \lambda}\right) \frac{d x^{\mu}}{d s} \frac{d x^{v}}{d s}
\end{aligned}
$$

For the non-relativistic case, considered here, we neglect the spatial coordinate derivatives compared to the time derivative, and take into account a stationary state, which means that the derivatives with time disappear. We obtain a particle accelerations proportional to the derivatives of the metric tensor element $g_{00}$ :

$$
a^{j}=\frac{d v^{j}}{d t}=-c^{2} g^{j \lambda} \frac{1}{2}(\underbrace{g_{\lambda 0,0}}_{0}+\underbrace{g_{\lambda 0,0}}_{0}-g_{00, \lambda}) \underbrace{\frac{d x^{0}}{d s}}_{1} \underbrace{\frac{d x^{0}}{d s}}_{1}=c^{2} g^{j \lambda} \frac{1}{2} g_{00, \lambda}
$$

which means that this matrix element behaves as a potential. Considering the Newtonian potential $V$ in this matric element,

$$
\begin{aligned}
& g_{00}=1-2 V \\
& V=-\frac{m}{r}, \quad[m]=1 m
\end{aligned}
$$

we obtain

$$
\begin{aligned}
& a^{j}=c^{2} g^{j \lambda} \frac{1}{2} \frac{\partial}{\partial x^{\lambda}}(1-2 V)=-c^{2} g^{j \lambda} \frac{\partial V}{\partial x^{\lambda}}= \\
& -c^{2} g^{j \lambda} \frac{\partial}{\partial x^{\lambda}}\left(\frac{m}{r}\right)=-m c^{2} g^{j 1} \frac{\partial}{\partial r}\left(\frac{1}{r}\right)
\end{aligned}
$$

With the Schwarzschild solution for the contravariant metric elements conjugated to the spatial coordinates, we obtain the three components of the acceleration,

$$
\begin{aligned}
& a^{1}=-\left(1-\frac{2 m}{r}\right)^{-1} \frac{m c^{2}}{r^{2}} \\
& a^{2}=0 \\
& a^{3}=0
\end{aligned}
$$

We notice that, for a rather small distance $r$, we obtain a correction of the Newtonian force, increasing the gravitational attraction.

\section{Quantum Particle as a Distribution of Matter}

We notice that, in this framework, a particle is conceived as a distribution of matter with a matter velocity field:

$$
v^{j}=\frac{\mathrm{d} x^{j}}{\mathrm{~d} t}=c \frac{\mathrm{d} x^{j}}{\mathrm{~d} s}=c \dot{x}^{j}
$$

From the relativistic quantum principle of the space-time interval invariance, 


$$
d s=\sqrt{g_{\mu \nu} d x^{\mu} d x^{v}}
$$

we obtain that the covariant derivative of velocity vector is perpendicular to this vector,

$$
\begin{aligned}
& 1=g_{\mu \nu} \dot{x}^{\mu} \dot{x}^{\nu} \\
& 0=\left(g_{\mu \nu} \dot{x}^{\mu} \dot{x}^{\nu}\right)_{: \sigma}=g_{\mu \nu}\left(\dot{x}^{\mu} \dot{x}_{: \sigma}^{v}+\dot{x}_{: \sigma}^{\mu} \dot{x}^{v}\right)=2 g_{\mu \nu} \dot{x}^{\mu} \dot{x}_{: \sigma}^{v} \\
& \dot{x}_{v} \dot{x}^{\nu}: \sigma=0
\end{aligned}
$$

If, besides the acceleration on a geodesic track, we consider an additional component $A^{\mu}$,

$$
\frac{d \dot{x}^{\mu}}{d s}=\dot{x}_{, v}^{\mu} \dot{x}^{v}=-\Gamma_{v \sigma}^{\mu} \dot{x}^{v} \dot{x}^{\sigma}+A^{\mu}
$$

we find that this component is perpendicular to the velocity vector:

$$
\begin{aligned}
& \left(\dot{x}_{, v}^{\mu}+\Gamma_{v \sigma}^{\mu} \dot{x}^{\sigma}\right) \dot{x}^{v}=A^{\mu} \\
& \dot{x}_{: v}^{\mu} \dot{x}^{v}=A^{\mu} \quad / \dot{x}_{\mu} \\
& \underbrace{\dot{x}_{\mu} \dot{x}_{: v}^{\mu}}_{0} \dot{x}^{v}=A^{\mu} \dot{x}_{\mu} \\
& \dot{x}_{\mu} A^{\mu}=0
\end{aligned}
$$

This means that any additional acceleration to the acceleration on a geodesic is perpendicular to the wave velocity, which is in agreement with wave propagation (Figure 5).

In particle propagation on a geodesic, other matter propagations are allowed only in perpendicular directions on the particle velocity.

We consider a quantum particle as a normalized matter distribution - a normalized integral of the matter density, in Cartesian coordinates,

$$
\int \rho(x, y, z, t) d x d y d z=\int|\psi(x, y, z, t)|^{2} d x d y d z=1
$$

or

$$
\int \rho\left(x^{i}, t\right) \mathrm{d} x^{1} \mathrm{~d} x^{2} \mathrm{~d} x^{3}=\int\left|\psi\left(x^{i}, t\right)\right|^{2} \frac{\partial(x, y, z)}{\partial\left(x^{1}, x^{2}, x^{3}\right)} \mathrm{d} x^{1} \mathrm{~d} x^{2} \mathrm{~d} x^{3}=1
$$

in curvilinear coordinates. We integrate the matter density

$$
\rho\left(x^{i}, t\right)=\left|\psi\left(x^{i}, t\right)\right|^{2} \frac{\partial(x, y, z)}{\partial\left(x^{1}, x^{2}, x^{3}\right)}
$$

on a space-time volume $\mathcal{V}$, in two systems of coordinates.

$$
\int_{\mathcal{V}} \rho\left(x^{\mu^{\prime}}\right) \mathrm{d} x 0^{0^{\prime}} \mathrm{d} x^{1^{\prime}} \mathrm{d} x^{2^{\prime}} \mathrm{d} x^{3^{\prime}}=\int_{\mathcal{V}} \rho\left(x^{\mu}\right) J \mathrm{~d} x^{0} \mathrm{~d} x^{1} \mathrm{~d} x^{2} \mathrm{~d} x^{3}
$$

and remark that the elements of the Jacobian,

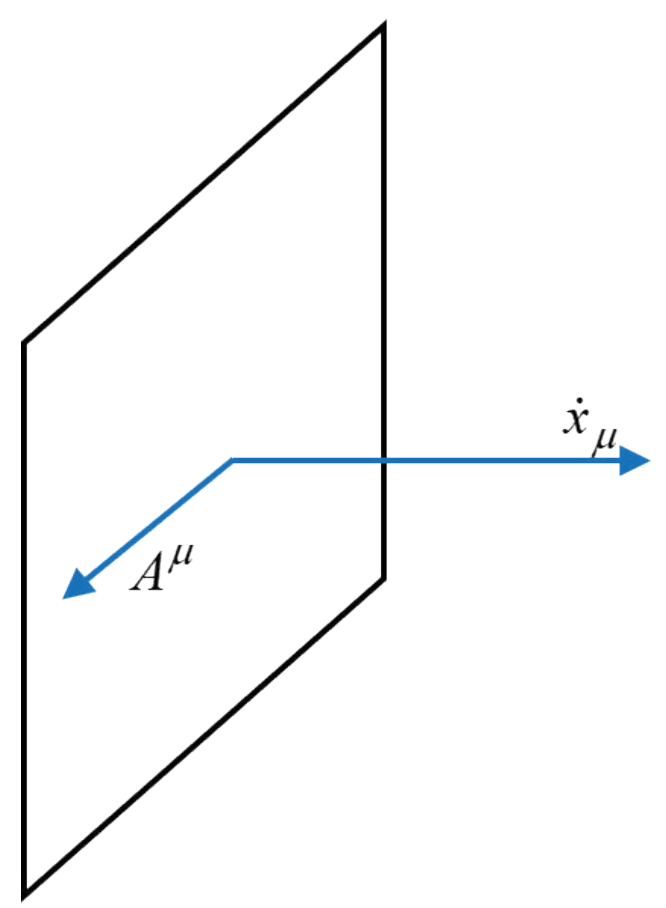

Figure 5. Plane wave-any additional particle acceleration $A^{\mu}$ to the acceleration on a geodesic is perpendicular to the velocity $\dot{x}_{\mu}$.

$$
J=\operatorname{Det}\left(J_{\mu^{\prime} \alpha}\right)=\operatorname{Det}\left(x_{, \alpha}^{\mu^{\prime}}\right)=\frac{\partial\left(x^{0^{\prime}}, x^{1^{\prime}}, x^{2^{\prime}}, x^{3^{\prime}}\right)}{\partial\left(x^{0}, x^{1}, x^{2}, x^{3}\right)}
$$

are also elements of a tensor transform, which, for the metric tensor is:

$$
g_{\alpha \beta}=\underbrace{x_{, \alpha}^{\mu^{\prime}}}_{J_{\mu^{\prime} \alpha}} \underbrace{x_{, \beta}^{v^{\prime}}}_{J_{v^{\prime} \beta}} g_{\mu^{\prime} v^{\prime}}
$$

Calculating the determinants,

$$
g=\operatorname{Det}\left(g_{\alpha \beta}\right)=J^{2} g^{\prime}
$$

we obtain the Jacobian as a function of the determinants of the metric tensor in the two systems of coordinates,

$$
J=\frac{\sqrt{-g}}{\sqrt{-g^{\prime}}}
$$

Introducing this expression of the Jacobian in the integral equation (9.9), we obtain an invariant for the matter density:

$$
\rho\left(x^{\alpha}\right) \sqrt{-g}=\rho\left(x^{\mu^{\prime}}\right) \sqrt{-g^{\prime}}=\text { Invariant }
$$

We consider a matter flow density $J^{\mu}$, as the product of the matter density with the velocity

$$
J^{\mu}=\rho \dot{x}^{\mu}
$$

and the matter conservation as a null covariant divergence

$J_{: \mu}^{\mu}=J_{, \mu}^{\mu}+\Gamma_{v \mu}^{\mu} J^{v}=J_{, v}^{v}+\Gamma_{v \mu}^{\mu} J^{v}=0$

From the general expression of the Christoffel symbol of the 
second kind as a function of the metric tensor,

$$
\Gamma_{v \sigma}^{\mu}=g^{\mu \lambda} \Gamma_{\lambda v \sigma}=g^{\mu \lambda} \frac{1}{2}\left(g_{\lambda v, \sigma}+g_{\lambda \sigma, v}-g_{\sigma v, \lambda}\right)
$$

we obtain

$\left.\Gamma_{v \mu}^{\mu}=\frac{1}{2} \underline{\underline{g^{\mu \lambda}}} \underline{\left(g_{\lambda v, \mu}\right.}+g_{\lambda \mu, v}-\underline{g_{\mu v, \lambda}}\right)=\frac{1}{2} g^{\mu \lambda} g_{\lambda \mu, v}=\frac{1}{2} g^{-1} g_{, v}=\frac{1}{2(-g)}(-g)_{, v}$

At the same time,

$$
(\sqrt{-g})_{, V}=\frac{1}{2 \sqrt{-g}}(-g)_{, v}
$$

which is

$$
1=\frac{1}{2 \sqrt{-g}} \frac{(-g)_{, v}}{(\sqrt{-g})_{, v}}
$$

From (9.18) and (9.19), we obtain the Christoffel symbol of the second kind in the expression (9.16),

$$
\Gamma_{\mu}^{\mu}=\frac{(\sqrt{-g})_{, \nu}}{\sqrt{-g}}
$$

which takes the form of a divergence of the product of the matter flow density with the square root of the metric determinant,

$$
J^{\mu}: \mu \sqrt{-g}=J_{, v}^{v} \sqrt{-g}+J^{v}(\sqrt{-g})_{, v}=\left(J^{v} \sqrt{-g}\right)_{, v}=\left(J^{\mu} \sqrt{-g}\right)_{, \mu}=0
$$

Integrating this expression on a volume $V$ of spatial coordinates,

$$
\int_{V}\left(J^{\mu} \sqrt{-g}\right), \mu \mathrm{d}^{3} x=0
$$

and separating the time derivative from the coordinate derivatives, we find a conservation law, as a matter variation in a volume $V$ by the flow of this matter through the surface $\Sigma_{\mathrm{V}}$ of the volume $V$ :

$$
\begin{aligned}
\left(\int_{V} J^{0} \sqrt{-g} \mathrm{~d}^{3} x\right)_{, 0} & =-\int_{V}\left(J^{m} \sqrt{-g}\right)_{, m} \mathrm{~d}^{3} x \\
& =-\oint_{\Sigma_{\mathrm{V}}} J^{m} \sqrt{-g} \mathrm{~d}^{2} x_{m}, \quad m=1,2,3
\end{aligned}
$$

In the nonrelativistic case, $\dot{x}^{m}<c$, weak gravitational field, we obtain

$$
\begin{aligned}
& J^{0}=\rho \dot{x}^{0} \approx \rho \\
& J^{m}=\rho \dot{x}^{m}
\end{aligned}
$$

while the conservation equation (9.23) takes a more explicit form, of density and matter flow,

$$
\rho_{, 0}=-\left(\rho \dot{x}^{m}\right)_{m}
$$

\section{Conclusion}

We discovered that a packet of Schrödinger wave functions, representing a quantum particle, is not in agreement with the Hamilton equations which describe the dynamics of such a particles - such an agreement is obtained only when instead of the Hamiltonian we consider the Lagrangian function. For a realistic particle, which must have a finite spectrum, we considered the relativistic Lagrangian, with a cut-off velocity $c$. We defined a Relativistic Quantum Principle: The time-dependent phase of a quantum particle is invariant to any change of coordinates. We obtained a wave equation for a quantum particle, depending on velocity, while the conventional Schrödinger equation describes only the amplitude of the particle wave-function - it includes a rapidly varying factor, with a phase proportional to the particle rest mass. On this basis, the relativistic kinematics and dynamics, the electromagnetic field equations, the particle spin, and the Schwarzschild-Newtonian dynamics in a gravitational field have been obtained. A quantum particle has been described by a wave function representing a distribution of conservative matter in motion, according to the General Theory of Relativity.

\section{References}

1. Brody T. The philosophy behind physics. Berlin and Heidelberg: Springer-Verlag. 1993.

2. Rowlands P. The foundations of physical law. New Jersey, London, Singapore, Beijing, Shanghai, Hong Kong, Taipei and Chennai: World Scientific. 2015.

3. Barret R, Delsanto PP, Tartaglia A. Physics: The ultimate adventure. Springer. 2016.

4. Cox B, Forshaw J. The quantum universe. Boston: Da Capo Press. 2012.

5. Penrose R. The road to reality. London: Vintage Books, 2004.

6. Hall MJW, Reginatto M. Ensembles on configuration space - Classical, quantum and beyond. In Fundamental Theories of Physics. Springer. 2016;184.

7. Feynman RP, Leighton RB, Sands M. The Feynman lectures on physics. Reading, Massachusetts: Addison-Wesley. 1963.

8. Landau L, Lifchitz E. Théorie du Champ. Moscou: Edition Mir. 1966.

9. Broglie L. Théorie de la quantification dans la nouvelle Mécanique. Paris: Hermann et Cie. 1932.

10. Heisenberg W. The physical principles of the quantum theory. New York: Dover Publications. 1949.

11. Pagels HR. The cosmic code - Quantum physics as the language of nature. Toronto, New York, London, Sydney: Bantam Books. 1983.

12. Stefanescu E. The relativistic dynamics as a quantum effect. J Basic Appl Res Int. 2014:1(1);13-23.

13. Stefanescu E. Open quantum physics and environmental heat conversion into usable energy. Sharjah (UAE), Brussels 
and Danvers (Massachusetts, USA): Bentham Science Publishers. 2014

14. Stefanescu E. Open quantum physics and environmental heat conversion into usable energy. Sharjah, U.A.E.: Bentham Science Publishers. 2017.

15. Kregar A. Aharonov-Bohm effect. University of Ljubljana. Faculty of Mathematics and Physics. Department of Physics. Seminar 4. Ljubljana. 2011.

16. Dirac PAM. General theory of relativity. New York, London, Sydney and Toronto: John Wiley \& Sons. 1975.

\section{*Correspondence to:}

Eliade Stefanescu

Advanced Studies in Physics Centre of the Romanian Academy

Academy of Romanian Scientists Bucharest

Romania

Tel: +4021-3188106/3138

E-mail: eliadestefanescu@yahoo.fr 